\title{
A GENERALIZATION OF BRØNDSTED'S RESULTS AND ITS APPLICATIONS
}

\author{
NORIKO MIZOGUCHI
}

(Communicated by Palle E. T. Jorgensen)

\begin{abstract}
We generalize Brøndsted's results in [2] and [3] in order to obtain uniform space versions of Caristi's fixed point theorem, Ekeland's variational principle and the drop theorem. Moreover, it is applied to weak convergence of random iterations.
\end{abstract}

\section{INTRODUCTION}

Ekeland [10] proved a useful theorem on complete metric spaces called the variational principle which has many applications in nonlinear analysis. Also, a fixed point theorem of different type from the previous ones was given on complete metric spaces by Caristi [7] and later seen to be equivalent to Ekeland's variational principle. In [2] and [3], Brøndsted obtained generalizations of these two theorems by introducing an ordering. Further, Penot [11] showed the equivalence of Ekeland's variational principle and a geometrical result on Banach spaces known as the drop theorem due to Daneš (see [8] and [9]).

On the other hand, Amemiya and Ando [1] proved weak convergence of random iterations drawn from metric projections onto linear subspaces $C_{0}, C_{1}$, $\cdots, C_{N}$ in Hilbert space. Similar results were obtained by Browder and Petryshin [4] and Bruck and Reich [6] on the assumption of recurrence, selection or periodicity. More generally, Bruck [5] considered under what conditions such an iteration converges when $C_{0}, C_{1}, \cdots, C_{N}$ are closed convex subsets of $H$.

In this paper, Brøndsted's results are generalized at first. Using it, we obtain extensions of Caristi's fixed point theorem and Ekeland's variational principle to uniform spaces, and of the drop theorem to locally convex spaces. As another application, we consider weak convergence of random iterations.

Received by the editors February 23, 1989.

1980 Mathematics Subject Classification (1985 Revision). Primary 47H10, 49A52.

Key words and phrases. Ekeland's variational principle, Caristi's fixed point theorem, drop, random iteration. 


\section{A generalization of BRøNDSTED's RESUlts}

Let $\preceq$ be an order relation on a topological space $X$. For $x \in X$ we denote by $S(x)$ the set of points $y \in X$ such that $x \preceq y$. It is said that a totally ordered subset $X_{0}$ of $X$ converges to some $x \in X$ provided the net $\left\{x_{\alpha}\right\}_{\alpha \in I}$ converges to $x \in X$ when the points of $X_{0}$ are indexed by the elements of a totally ordered set $(I, \preceq)$ so that for $\alpha, \beta \in I, x_{\alpha} \preceq x_{\beta}$ if and only if $\alpha \preceq \beta$.

Lemma. Let $X$ be a complete uniform space with a family $\left\{d_{\lambda}\right\}_{\lambda \in \Lambda}$ of pseudometrics inducing the topology of $X, \preceq$ be an ordering on $X$ and $\left\{f_{\lambda}\right\}_{\lambda \in \Lambda}$ be a family of real-valued functions on $X$ satisfying

(i) $f_{\lambda}$ is nonincreasing and bounded below for each $\lambda \in \Lambda$;

(ii) For each $\lambda \in \Lambda$ and any $\varepsilon>0$, there exists $\delta_{\lambda}>0$ such that $x \preceq y$ and $f_{\lambda}(x)-f_{\lambda}(y)<\delta_{\lambda}$ imply $d_{\lambda}(x, y)<\varepsilon$.

Then an arbitrary totally ordered subset of $X$ converges to some point in $X$. Furthermore, if the following condition

(iii) $S(x)$ is a closed subset of $X$ for each $x \in X$, holds, then the limit point of each maximal totally ordered subset of $X$ is a maximal element in $X$.

Proof. Let $\left\{x_{\alpha}\right\}_{\alpha \in I}$ be a totally ordered subset of $X$ indexed by the previous method. Fix an arbitrary $\lambda \in \Lambda$. Since $f_{\lambda}$ is nonincreasing and bounded below, the net $\left\{f_{\lambda}\left(x_{\alpha}\right)\right\}_{\alpha \in I}$ converges in $\mathbf{R}$ and hence, given $\varepsilon>0$ and a corresponding $\delta_{\lambda}>0$ as in (ii), there is $\alpha(\lambda) \in I$ satisfying

$$
0 \leq f_{\lambda}\left(x_{\beta}\right)-f_{\lambda}\left(x_{\gamma}\right)<\delta_{\lambda} \quad \text { for } \gamma \succeq \beta \succeq \alpha(\lambda) .
$$

Then we have $d_{\lambda}\left(x_{\beta}, x_{\gamma}\right)<\varepsilon$ for $\gamma \succeq \beta \succeq \alpha(\lambda)$ from (ii). This implies that $\left\{x_{\alpha}\right\}_{\alpha \in I}$ is a Cauchy net and therefore convergent in $X$ by the completeness of $X$. Now suppose $\left\{x_{\alpha}\right\}_{\alpha \in I}$ is a maximal totally ordered subset of $X$. Under the hypothesis (iii), the limit point $z$ of $\left\{x_{\alpha}\right\}_{\alpha \in I}$ belongs to the closed set $S\left(x_{\alpha}\right)$, that is, $x_{\alpha} \preceq z$ for each $\alpha \in I$. The maximality of $z$ follows from that of $X_{0}$.

Note that there always exists a maximal totally ordered subset of $X$ by Zorn's Lemma. This result is a slight generalization of those of Brøndsted in [2] and [3] but much more convenient and natural than his. For example, our lemma is applicable to a fixed point theorem for a contraction mapping $T: X \rightarrow X$, i.e. $d_{\lambda}(T x, T y) \leq k_{\lambda} d_{\lambda}(x, y)$ for all $x, y \in X$ and $\lambda \in \Lambda$, where $k_{\lambda}$ is a constant dependent on $\lambda$ with $0 \leq k_{\lambda}<1$. However, it is not clear how one would deduce such a fixed point theorem from Brøndsted's results.

\section{Extensions of Results of Caristi, Ekeland and DaneŠ}

We state extended versions of Caristi's fixed point theorem ([7]), Ekeland's variational principle ([10]) and the drop theorem ([8]) in uniform or locally convex spaces. 
Theorem 1 (extended Caristi's fixed point theorem). Let $X$ be a complete uniform space with a family $\left\{d_{\lambda}\right\}_{\lambda \in \Lambda}$ of pseudometrics inducing the topology of $X$, $f: X \rightarrow \mathbf{R} \cup\{+\infty\}$ be a lower semicontinuous, bounded below and proper (i.e. not identically $+\infty)$ function and $\left\{\nu_{\lambda}\right\}_{\lambda \in \Lambda}$ be a family of positive numbers. If $T: X \rightarrow 2^{X}$ has the property that, for any $\lambda \in \Lambda$,

$$
\nu_{\lambda} d_{\lambda}(x, y)+f(y) \leq f(x) \quad \text { for each } x \in X \text { and } y \in T x,
$$

then there exists $z \in X$ with $T z=\{z\}$.

Theorem 2 (extended Ekeland's variational principle). Let $X,\left\{d_{\lambda}\right\}_{\lambda \in \Lambda}, f$ and $\left\{\nu_{\lambda}\right\}_{\lambda \in \Lambda}$ be as in Theorem 1. Then for any $x_{0} \in X$, there exists $z \in X$ such that

(i) for each $x \in X$ with $x \neq z$, there is $\lambda \in \Lambda$ satisfying

$$
f(z)<f(x)+\nu_{\lambda} d_{\lambda}(z, x)
$$

(ii) for any $\mu \in \Lambda$,

$$
f(z) \leq f\left(x_{0}\right)-\nu_{\mu} d_{\mu}\left(z, x_{0}\right) .
$$

Let $E$ be a vector space and $p$ a seminorm on $E$. For $x \in E$ and $A$, $B \subset E$, we define

$$
p(x, A)=\inf \{p(x-a): a \in A\}
$$

and

$$
p(A, B)=\inf \{p(a-b): a \in A, b \in B\} .
$$

Assuming convexity of $A$, it follows that $p(\cdot, A): E \rightarrow \mathbf{R}$ is a convex function. For a convex subset $B$ of $E$ and $x \notin B$, we denote by $D(x, B)$ the convex hull of $B \cup\{x\}$ and call it the drop associated with $x$ and $B$. Note that if $B$ is a bounded, closed and convex subset of a topological vector space, so is the drop $D(x, B)$ for each $x \notin B$.

Theorem 3 (extended drop theorem). Let $E$ be a locally convex topological vector space whose topology is generated by a family $\left\{p_{\lambda}\right\}_{\lambda \in \Lambda}$ of seminorms on $E$. If $A$ is a complete subset of $E$ and $B$ is a closed, convex and bounded subset of $E$ with $p_{\lambda}(A, B)>0$ for any $\lambda \in \Lambda$, then, for each $x_{0} \in A$, there exists $z \in A \cap D\left(x_{0}, B\right)$ such that $A \cap D(z, B)=\{z\}$.

We deduce Theorem 1 from the Lemma and then prove the other theorems by showing that they are equivalent to Theorem 1 .

Proof of Theorem 1. Fix $x \in X$ with $f(x)<+\infty$. Putting $X^{\prime}=\{y \in X$ : $f(y) \leq f(x)\}, X^{\prime}$ is complete by the lower semicontinuity of $f$. Define an ordering on $X^{\prime}$ by $u \preceq v$ if and only if

$$
\nu_{\lambda} d_{\lambda}(u, v) \leq f(u)-f(v) \quad \text { for all } \lambda \in \Lambda .
$$

Applying the Lemma to $\left(X^{\prime}, \preceq\right)$ and $f_{\lambda}=f / \nu_{\lambda}$, we obtain a maximal element $z$ in $X$. This point $z$ is the desired one. 
Proof of "Theorem $1 \rightarrow$ Theorem 3". Given $x_{0} \in E$, we put $X=D\left(x_{0}, B\right) \cap A$. From the hypothesis, for each $\lambda$ we have $\delta_{\lambda}=p_{\lambda}(A, B)>0$ and there exists $M_{\lambda}>0$ with $p_{\lambda}(x-y) \leq M_{\lambda}$ for all $x \in X$ and $y \in B$. Define $T: X \rightarrow 2^{X}$ and a lower semicontinuous function $f: X \rightarrow \mathbf{R}$ by

$$
T x=D(x, B) \cap A
$$

and

$$
f(x)=\sup _{\lambda \in \Lambda} \frac{p_{\lambda}(x, B)}{M_{\lambda}}
$$

for all $x \in X$, respectively. Then it is seen that for all $\lambda \in \Lambda, x \in X$ and $y \in T x$,

$$
p_{\lambda}(y-x) \leq \frac{M_{\lambda}^{2}}{\delta_{\lambda}}\{f(x)-f(y)\} .
$$

Indeed, let $\lambda \in \Lambda$ and $x \in X$. For each $y \in T x$, take $z \in B$ and $t \in[0,1]$ with $y=(1-t) x+t z$. By the convexity of $B, p_{\lambda}(\cdot, B)$ is a convex real-valued function on $X$. It follows that

$$
\begin{aligned}
p_{\lambda}(y, B) & \leq(1-t) p_{\lambda}(x, B)+t p_{\lambda}(z, B) \\
& =(1-t) p_{\lambda}(x, B) .
\end{aligned}
$$

Thus we have

$$
f(y)=\sup _{\lambda \in \Lambda} \frac{p_{\lambda}(y, B)}{M_{\lambda}} \leq(1-t) \sup _{\lambda \in \Lambda} \frac{p_{\lambda}(x, B)}{M_{\lambda}}=(1-t) f(x),
$$

that is,

$$
t \leq \frac{f(x)-f(y)}{f(x)} \leq \frac{M_{\lambda}}{\delta_{\lambda}}\{f(x)-f(y)\} .
$$

Since $y-x=t(z-x)$,

$$
p_{\lambda}(y-x)=t p_{\lambda}(z-x) \leq t M_{\lambda} \leq \frac{M_{\lambda}^{2}}{\delta_{\lambda}}\{f(x)-f(y)\} .
$$

By Theorem 1 we obtain $z \in X$ satisfying $T z=\{z\}$, i.e.

$$
z \in D\left(x_{0}, B\right) \cap A \text { and } D(z, B) \cap A=\{z\} .
$$

Proof of "Theorem $3 \rightarrow$ Theorem 2". Fix $x_{0} \in X$. We may assume $f\left(x_{0}\right)<+\infty$ without any loss of generality. We may also suppose that each $d_{\lambda}$ is bounded on $X$ by replacing $d_{\lambda}$ with $d_{\lambda}^{\prime}$ if necessary, where

$$
d_{\lambda}^{\prime}(x, y)=\min \left\{\frac{f\left(x_{0}\right)-\inf f(x)+1}{\nu_{\lambda}}, d_{\lambda}(x, y)\right\} \quad \text { for } x, y \in X .
$$

In fact, it is clear from the definition of $d_{\lambda}^{\prime}$ that if the conclusion (i) in Theorem 2 holds for $d_{\lambda}^{\prime}$, then it holds for $d_{\lambda}$. Suppose that the conclusion (ii) in Theorem 2 holds for $d_{\mu}^{\prime}$, i.e.

$$
f\left(x_{0}\right)-f(z) \geq \nu_{\mu} d_{\mu}^{\prime}\left(x_{0}, z\right)
$$


Since

$$
f(z) \geq \inf f(X)>\inf f(X)-1,
$$

we have

$$
\frac{f\left(x_{0}\right)-f(z)}{\nu_{\mu}}<\frac{f\left(x_{0}\right)-\inf f(X)+1}{\nu_{\mu}} .
$$

It follows that

$$
d_{\mu}^{\prime}\left(x_{0}, z\right)<\frac{f\left(x_{0}\right)-\inf f(X)+1}{\nu_{\mu}}
$$

and hence $d_{\mu}^{\prime}\left(x_{0}, z\right)=d_{\mu}\left(x_{0}, z\right)$. Therefore we obtain

$$
f\left(x_{0}\right)-f(z) \geq \nu_{\mu} d_{\mu}\left(x_{0}, z\right) .
$$

Let $F$ be the Banach space of all bounded functions on $X$ with supremum norm $\|\cdot\|$ and $Y=\prod_{\lambda \in \Lambda}\left(F_{\lambda},\|\cdot\|_{\lambda}\right)$, where $F_{\lambda}=F$ and $\|\cdot\|_{\lambda}=\|\cdot\|$ for all $\lambda \in \Lambda$. Putting, for any $x \in X$ and $\lambda \in \Lambda$,

$$
d_{x}(\lambda)(y)=d_{\lambda}(x, y) \quad \text { for all } y \in X,
$$

we have $d_{x}(\lambda) \in(F,\|\cdot\|)$ and $d_{\lambda}(x, y)=\left\|d_{x}(\lambda)-d_{y}(\lambda)\right\|$. Thus we can identify $x \in X$ with $\left(x_{\lambda}\right)_{\lambda \in \Lambda} \in Y$, where $x_{\lambda}=d_{x}(\lambda)$. Let $E=Y \times \mathbf{R}$ and $\tau$ be the topology on $E$ induced by the family $\left\{p_{\lambda}\right\}_{\lambda \in \Lambda}$ of seminorms on $E$ defined, for each $\lambda \in \Lambda$, by

$$
p_{\lambda}(x, t)=\max \left\{|| x_{\lambda} \|,|t|\right\}
$$

for all $(x, t)=\left(\left(x_{\lambda}\right)_{\lambda \in \Lambda}, t\right) \in E$. Set $g=-f, m=\sup \{g(x): x \in X\}$ and $A=\{(x, t) \in X \times \mathbf{R}: t \leq g(x)\}$. Then $A$ is complete with respect to $\tau$ from the completeness of $X$ and the upper semicontinuity of $g$. We may assume that $x_{0}=0$ and $f\left(x_{0}\right)=g\left(x_{0}\right)=0$ by replacing $f$ with the function $h$ defined by

$$
h(x)=f\left(x_{0}+x\right)-f\left(x_{0}\right) \quad \text { for each } x \in X-x_{0} .
$$

Fix $r \in \mathbf{R}$ with $r>m$ and, for this $r$, let

$$
B=\left\{(x, r) \in E:\left\|x_{\lambda}\right\| \leq \frac{r}{\nu_{\lambda}} \text { for all } \lambda \in \Lambda\right\}
$$

and $K=\mathbf{R}^{+} B$. It follows that $B$ is a closed, convex and bounded subset of $E$ and $p_{\lambda}(B, A) \geq r-m>0$ for each $\lambda \in \Lambda$. Theorem 3 assures us of the existence of $(z, s) \in A \cap D((0,0), B)$ satisfying $A \cap D((z, s), B)=\{(z, s)\}$. Now, it is easily seen that

$$
K=\left\{(y, t) \in E: t \geq \nu_{\lambda}\left\|y_{\lambda}\right\| \text { for all } \lambda \in \Lambda\right\} .
$$

From $(z, s) \in D((0,0), B) \cap A \subset K \cap A$, we have

$$
g(z) \geq \nu_{\lambda}\left\|z_{\lambda}\right\|=\nu_{\lambda} d_{\lambda}\left(z, x_{0}\right) \quad \text { for each } \lambda \in \Lambda \text {. }
$$

Suppose $(x, t) \in(z, s)+K, x \in X, x \neq z$ and $t \leq m$; then $t-s \geq \nu_{\lambda}\left\|x_{\lambda}-z_{\lambda}\right\|$ for all $\lambda \in \Lambda$ and hence $t-s>0$. From $(x-z, t-s)=q(w, r-s)$, where $q=(t-s) /(r-s)$ and $w=(x-z) / q$, we get

$$
(w, r-s)=\frac{1}{q}(x-z, t-s) \in K \text {. }
$$


Note that $0<q<1$ since $0<t-s \leq m-s<r-s$. Since $K$ is a convex cone, we have

$$
(z+w, r)=(z, s)+(w, r-s) \in K
$$

so

$$
(z+w, r) \in K \cap(Y \times\{r\})=B .
$$

By the convexity of $D((z, s), B)$, we have

$$
(x, t)=(1-q)(z, s)+q(z+w, r) \in D((z, s), B) .
$$

This implies $(x, t) \notin A$. Thus for any $x \in X$ with $x \neq z$, we obtain $(x, g(x)) \notin(z, s)+K$, i.e.

$$
f(z)<f(x)+\nu_{\lambda} d_{\lambda}(x, z) \quad \text { for all } \lambda \in \Lambda .
$$

This completes the proof.

This proof is a modification (to accommodate a family of pseudometrics rather than a metric) of Penot's proof in [11] that the drop theorem implies Ekeland's variational principle.

It is immediate that Theorem 2 implies Theorem 1. These proofs also show the equivalences among Theorems 1,2 and 3 .

\section{CONVERGENCE OF RANDOM ITERATION}

We give another application of the lemma. Let $C$ be a closed convex subset of a Hilbert space $H$. A mapping $T: C \rightarrow C$ with a nonempty fixed-point set $F(T)$ is said to be quasi-nonexpansive provided $\|T x-f\| \leq\|x-f\|$ for all $x \in C$ and $f \in F(T)$. Note that $F(T)$ is a closed convex subset of $H$. In fact, let $f_{1}, f_{2} \in F(T), 0<t<1$ and $f=t f_{1}+(1-t) f_{2}$. Then we have

$$
\begin{aligned}
\left\|f_{1}-f\right\| & =\left\|f_{1}-f_{2}\right\|-\left\|f_{2}-f\right\| \\
& \leq\left\|f_{1}-f_{2}\right\|-\left\|f_{2}-T f\right\| \\
& \leq\left\|f_{1}-T f\right\| \\
& \leq\left\|f_{1}-f\right\|
\end{aligned}
$$

and hence

$$
\left\|f_{1}-f\right\|=\left\|f_{1}-T f\right\|
$$

Similarly we obtain

$$
\left\|f_{2}-f\right\|=\left\|f_{2}-T f\right\| .
$$

These two equalities imply that $T f=f$. Suppose that $\left\{T_{\alpha}\right\}_{\alpha \in I}$ is a family of quasi-nonexpansive mappings of $C$ into itself with a nonempty common fixed-point set $F$. A random iteration drawn from $\left\{T_{\alpha}\right\}_{\alpha \in I}$ means a sequence $\left\{x_{n}\right\}$ such that

$$
x_{0}=x \in C \quad \text { and } \quad x_{n}=T_{r(n)} x_{n-1} \quad \text { for } n \geq 1 \text {, where } r: \mathbf{N} \rightarrow I .
$$

We call $x \in F$ a directional interior point of $F$ for $y \in H$ with $\|y\|=1$ if there exists $\delta_{y}>0$ satisfying $x \pm \delta_{y} y \in F$. When the set $F$ has a directional 
interior point $f_{y}$ for each $y \in H$ with $\|y\|=1$, with corresponding $\delta_{y}>0$, an ordering $\preceq$ on $C$ is introduced for $u, v \in C$ by $u \preceq v$ if and only if

$$
2 \delta_{y}|\langle u-v, y\rangle| \leq\left\|u-f_{y}\right\|^{2}-\left\|v-f_{y}\right\|^{2}
$$

for all $y \in H$ with $\|y\|=1$. We use the lemma to establish the following in this situation.

Theorem 4. Let $C$ be a closed convex subset of a Hilbert space $H$ and $\left\{T_{\alpha}\right\}_{\alpha \in I}$ be a family of quasi-nonexpansive mappings of $C$ into itself. If the common fixed-point set $F$ of $\left\{T_{\alpha}\right\}_{\alpha \in I}$ has a directional interior point $f_{y}$ for each $y \in H$ with $\|y\|=1$, then for each $x \in C$, a random iteration $\left\{x_{n}\right\}$ drawn from $\left\{T_{\alpha}\right\}_{\alpha \in I}$ weakly converges to some $z \in C$. Further, if the orbit $\left\{x_{n}\right\}$ is a maximal totally ordered subset of $C$ with respect to the ordering $\preceq$ defined above, then the limit point $z$ belongs to $F$.

Proof. From the quasi-nonexpansiveness of $T_{\alpha}$, it follows that

$$
\left\|T_{\alpha} x-\left(f_{y} \pm \delta_{y} y\right)\right\| \leq\left\|x-\left(f_{y} \pm \delta_{y} y\right)\right\| .
$$

By a simple calculation, we have

$$
2 \delta_{y}\left|\left\langle x-T_{\alpha} x, y\right\rangle\right| \leq\left\|x-f_{y}\right\|^{2}-\left\|T_{\alpha} x-f_{y}\right\|^{2}
$$

for each $y \in H$ with $\|y\|=1$, which means $x \leq T_{\alpha} x$. Since the function $x \rightarrow\left\|x-f_{y}\right\|^{2}$ is weakly lower semicontinuous, it is sufficient to apply the lemma to the totally ordered set $\left\{x_{n}\right\}$.

Remark. Let $C,\left\{T_{\alpha}\right\}_{\alpha \in I}$ and $F$ be as stated above. If the interior of $F$ is nonempty, there exist $f \in F$ and $\delta>0$ such that

$$
2 \delta\|x-T x\| \leq\|x-f\|^{2}-\|T x-f\|^{2} \quad \text { for all } x \in C .
$$

Then defining an ordering $\preceq$ for $u, v \in C$ by $u \preceq v$ if and only if

$$
2 \delta\|u-v\| \leq\|u-f\|^{2}-\|v-f\|^{2},
$$

we have a result for strong convergence of random iterations similar to Theorem 4.

\section{ACKNOWLEDGMENTS}

The author wishes to express her hearty thanks to Professor W. Takahashi for many kind suggestions and advice in the course of preparing the present paper, and also to the referee for his many helpful suggestions.

\section{REFERENCES}

1. I. Amemiya and T. Ando, Convergence of random products of contractions in Hilbert space, Acta Sci. Math. (Szeged) 26 (1965), 239-244.

2. A. Brøndsted, On a lemma of Bishop and Phelps, Pacific J. Math. 55 (1974), 335-341.

3. __ Common fixed points and partial orders, Proc. Amer. Math. Soc. 77 (1979), 365-368. 
4. F. E. Browder and W. V. Petryshin, Construction of fixed points of nonlinear mappings in Hilbert spaces, J. Math. Anal. Appl. 20 (1967), 197-228.

5. R. E. Bruck, Random products of contractions in metric and Banach spaces, J. Math. Anal. Appl. 88 (1982), 319-332.

6. R. E. Bruck and S. Reich, Nonexpansive projections and resolvents of accretive operators in Banach spaces, Houston J. Math. 3 (1977), 459-470.

7. J. Caristi, Fixed point theorems for mappings satisfying inwardness conditions, Trans. Amer. Math. Soc. 215 (1976), 241-251.

8. J. Daneš, A geometric theorem useful in nonlinear functional analysis, Boll. Un. Mat. Ital. 6 (1972), 369-375.

9. __ Equivalence of some geometric and related results of nonlinear functional analysis, Comment. Math. Univ. Carolinae 26 (1985), 443-454.

10. I. Ekeland, On the variational principle, J. Math. Anal. Appl. 47 (1974), 324-353.

11. J. P. Penot, The drop theorem, the petal theorem and Ekeland's variational principle, Nonlinear Anal. 10 (1986), 813-822.

Department of Information Science, Tokyo Institute of Technology, Oh-okayama, MEgURO-KU, TOKYO 152, JAPAN 